\title{
European Union standard for tuberculosis care on treatment of multidrug-resistant tuberculosis following new World Health Organization recommendations
}

To the Editor:

The European Respiratory Journal recently published a European Respiratory Society (ERS) and European Centre for Disease Prevention and Control (ECDC) statement that provided a 2017 update of the European Union Standards for Tuberculosis Care (ESTC) [1], resulting from a joint effort of the ERS and the ECDC.

A panel of international experts coordinated by ERS and ECDC updated the ESTC document released in $2012[2,3]$. The structure and principles of the patient-centred ESTC remained the same, including 21 standards in the areas of tuberculosis (TB) diagnosis, treatment, HIV and comorbidities (activity coordinated by ERS), as well as in public health and prevention (activity coordinated by ECDC). The ESTC document, which aims to be an easy-to-use resource, calls on both clinicians and public health workers to provide the best possible diagnosis, treatment and prevention of TB.

On August 17, 2018, the World Health Organization (WHO) released an important rapid communication document, "Key changes to treatment of multidrug- and rifampicin-resistant tuberculosis" [4]. This document was published ahead of more detailed WHO policy guidelines on multidrug-resistant TB (MDR-TB) treatment, which are expected to be published by the end of 2018. In summary, the WHO document recommends a revised grouping of the drugs used to treat MDR-TB and new information to design an adequate regimen. Among the key changes are the inclusion of fluoroquinolones, bedaquiline and linezolid in group A (to be prioritised when designing an MDR-TB regimen), while second-line injectables are now in group $\mathrm{C}$ because of safety concerns (notably only amikacin and streptomycin are now recommended) (table 1) [4].

This WHO publication renders obsolete all existing guidelines and standards that were based on the 2016 WHO MDR-TB guidelines [5], including the text of the European Union-specific requirements of standard 12 in the ESTC 2017 update [1]. While the standard as such is still valid, this section is no longer up to date. The specific concerned text is the following:

The individualised regimen should include at least five effective TB medicines during the intensive phase, including pyrazinamide and four core second-line TB medicines. Drugs should be chosen as follows: one chosen from group A, one from group B, and at least two from group C (table 3). If the minimum number of five effective TB medicines cannot be composed from drugs included in groups $\mathrm{A}$ to $\mathrm{C}$, an agent from group D2 and other agents from group D3 may be added to bring the total to five. If pyrazinamide cannot be used (e.g. due to resistance or toxicity) an additional agent from group $\mathrm{C}$ or $\mathrm{D}$ can be added to strengthen the regimen. Total treatment duration ranges from 20 to 24 months, with the recommended intensive phase being 8 months [6].

Based on the newly published WHO document, the text needs to read as follows, complemented by table 1 (as a substitution for table 3 in the ESTC 2017 update [1]):

@ERSpublications

The key changes to treatment of multidrug-resistant tuberculosis (World Health Organization, August 2018) should be taken into account when applying standard 12 of the European Union Standards for Tuberculosis Care http://ow.ly/nVwl30lisaF

Cite this article as: Migliori GB, Sotgiu G, Rosales-Klintz S, et al. European Union standard for tuberculosis care on treatment of multidrug-resistant tuberculosis following new World Health Organization recommendations. Eur Respir J 2018; 52: 1801617 [https://doi.org/10.1183/13993003.016172018]. 


\begin{tabular}{|c|c|c|c|}
\hline Group & & Medicine & Abbreviation \\
\hline \multirow[t]{3}{*}{ A } & \multirow{3}{*}{$\begin{array}{l}\text { Include all three medicines } \\
\text { (unless they cannot be used) }\end{array}$} & Levofloxacin OR moxifloxacin & Lfx/Mfx \\
\hline & & Bedaquiline & $\mathrm{Bdq}$ \\
\hline & & Linezolid & Lzd \\
\hline \multirow[t]{2}{*}{ B } & \multirow{2}{*}{$\begin{array}{l}\text { Add both medicines } \\
\text { (unless they cannot be used) }\end{array}$} & Clofazimine & $\mathrm{Cfz}$ \\
\hline & & Cycloserine OR terizidone & $\mathrm{Cs} / \mathrm{Trd}$ \\
\hline \multirow[t]{7}{*}{ C } & \multirow{7}{*}{$\begin{array}{l}\text { Add to complete the regimen and } \\
\text { when medicines from groups A } \\
\text { and B cannot be used }\end{array}$} & Ethambutol & $E$ \\
\hline & & Delamanid & $\mathrm{Dlm}$ \\
\hline & & Pyrazinamide & Z \\
\hline & & Imipenem-cilastatin OR meropenem & Ipm-Cln/Mpm \\
\hline & & Amikacin (OR streptomycin) & Am (S) \\
\hline & & Ethionamide OR prothionamide & Eto/Pto \\
\hline & & $p$-aminosalicylic acid & PAS \\
\hline
\end{tabular}

Reproduced and modified from [4] with permission.

Individualised MDR-TB regimens for adults and children should follow the priority ranking of recommended medicines. An adequate regimen is designed by adding medicines sequentially in descending order as they are listed in the three groups: 1) include all three medicines from group A (unless they cannot be used); 2) add both medicines from group B (unless they cannot be used); and 3) add medicines from group $\mathrm{C}$ to complete the regimen when medicines from groups $\mathrm{A}$ and $\mathrm{B}$ cannot be used. The new WHO publication states: "Longer MDR-TB regimens usually last 1820 months and may be standardized or individualized. These regimens are usually designed to include at least five medicines considered to be effective" [4].

Complete details on the number of drugs and the overall treatment duration will be provided in the forthcoming consolidated, updated and more detailed WHO policy guidelines on MDR-TB treatment. We propose that while waiting for the final and complete version of the document, the revised version of text for standard 12, as suggested here, is adopted as the European Union-specific requirement.

Giovanni Battista Migliori $\oplus^{1}$, Giovanni Sotgiu $\oplus^{2}$, Senia Rosales-Klintz ${ }^{3}$ and Marieke J. van der Werf $\oplus^{3}$

${ }^{1}$ Istituti Clinici Scientifici Maugeri IRCCS, Tradate, Italy. ${ }^{2}$ Clinical Epidemiology and Medical Statistics Unit, Dept of Biomedical Sciences, University of Sassari, Sassari, Italy. ${ }^{3}$ European Centre for Disease Prevention and Control, Stockholm, Sweden.

Correspondence: Marieke J. van der Werf, European Centre for Disease Prevention and Control (ECDC), Gustav den III:s Boulevard 40, 16973 Solna, Sweden. E-mail: Marieke.vanderwerf@ecdc.europa.eu

Received: Aug 242018 | Accepted: Aug 242018

Conflict of interest: None declared.

\section{References}

1 Migliori GB, Sotgiu G, Rosales-Klintz S, et al. ERS/ECDC Statement: European Union standards for tuberculosis care, 2017 update. Eur Respir J 2018; 51: 1702678.

2 Migliori GB, Zellweger JP, Abubakar I, et al. European Union Standards for Tuberculosis Care. Eur Respir J 2012; 39: 807-819.

3 van der Werf MJ, Sandgren A, D'Ambrosio L, et al. The European Union standards for tuberculosis care: do they need an update? Eur Respir J 2014; 43: 933-942.

4 WorldHealth Organization. Rapid Communication: Key changes to treatment of multidrug- and rifampicin-resistant tuberculosis (MDR/RR-TB). Geneva, World Health Organization, 2018. Available from: www. who.int/tb/publications/2018/rapid_communications_MDR/en/

5 World Health Organization. WHO treatment guidelines for drug-resistant tuberculosis: 2016 update. Geneva, World Health Organization, 2016.

6 World Health Organization. Compendium of WHO guidelines and associated standards: ensuring optimum delivery of the cascade of care for patients with tuberculosis. Geneva, World Health Organization, 2017.

The content of this work is copyright of the authors or their employers. Design and branding are copyright (CERS 2018. 